\title{
Using Interactive Visual Analytics to Optimize in Real-Time Blood Products Inventory at a Blood Bank
}

\author{
Jaber RAD ${ }^{\mathrm{a}}$, Jason G. QUINN ${ }^{\mathrm{b}}$, Calvino CHENG ${ }^{\mathrm{b}}$, Robert LIWSKI ${ }^{\mathrm{b}}$, \\ Samina Raza ABIDI ${ }^{\mathrm{a}, \mathrm{c}}$ and Syed Sibte Raza ABIDI ${ }^{\mathrm{a}, 1}$ \\ ${ }^{a}$ NICHE Research Group, Faculty of Computer Science, Dalhousie University, \\ Halifax, Canada \\ ${ }^{\mathrm{b}}$ Department of Pathology and Laboratory Medicine, Nova Scotia Health \\ Authority, Halifax, Canada \\ ${ }^{\mathrm{c}}$ Department of Community Health and Epidemiology, Dalhousie University, \\ Halifax, Canada
}

\begin{abstract}
Blood products and their derivatives are perishable commodities that require an efficient inventory management to ensure both a low wastage rate and a high product availability rate. To optimize blood product inventory, blood transfusion services need to reduce wastage by avoiding outdates and improve availability of different blood products. We used advance visualization techniques to design and develop a highly interactive real-time web-based dashboard to monitor the blood product inventory and the on-going blood unit transactions in near-realtime based on analysis of transactional data. Blood transfusion staff use the dashboard to locate units with specific characteristics, investigate the lifecycle of the units, and efficiently transfer units between facilities to minimize outdates.
\end{abstract}

Keywords. Interactive Visual Analytics, Data Visualization, Blood Transfusion Services, Dashboard, Inventory Management

\section{Introduction}

Blood product inventory management is a highly complex operation that relies on interpretation of multi-dimensional data including lifecycle-dependent variables such as real-time transactional status of the units, age of the units, discard reasons and lifecycleindependent variables such as blood types, special attributes (e.g., irradiation and phenotyping) and the suppliers [1]. Currently, hospitals and blood suppliers employ a mixture of non-standardized methodologies including paper reporting, spreadsheets, adhoc laboratory information system queries, and often the data is used for month-end reporting rather than near-real-time decision-making. This month-end reporting consists of the summarized data and therefore it cannot be used for auditing causes of inefficiencies. Although the raw data gives a high resolution of the blood product inventory, the interpretive richness and timeliness of the raw data is lost, and quality improvement, such as waste reduction and efficient utilization, is not possible.

${ }^{1}$ Corresponding Author, Syed Sibte Raza Abidi, Faculty of Computer Science, Dalhousie University, 6050 University Avenue, Halifax, NS B3H 4R2, Canada; E-mail: ssrabidi@dal.ca. 
Interactive Visual Analytics (IVA) provides a suite of data visualizations to interpret and interact with high-volume and high-dimensional data. Operational dashboards are being used in various domains to provide an easy-to-interpret overview of on-going processes, activities, users and inventories for system management, diagnostics and optimization. Given the rapid transactions taking place in a blood bank, Blood Transfusion Services (BTS) need an up-to-date account of blood products within their inventory to (a) ensure there is adequate supply of blood products to meet demand; (b) minimize blood unit's wastage due to expiry; and (c) find matching blood units in response to specialized transfusion criteria and demand from medical units.

In this paper, we present an innovative Blood Inventory Dashboard (BID) to help blood transfusion services visualize in real-time the evolving inventory of the blood bank, to help improve efficiency and reduce wastage. BID provides the monitoring of three blood products - i.e., Red Blood Cells (RBC), Platelets and Plasma. Using advanced IVA methods, we have developed and applied data visualizations for (a) real-time management of the blood bank's inventory; and (b) auditing the blood unit distribution process to identify reasons/processes leading to inefficiencies and wastage. BID analyzes streams of blood transaction data that constitutes the unit's lifecycle and characteristics/product type and location within the hospital. BID presents the real-time inventory in an interactive format for transfusion services to know the volume of specific blood units and prioritize units for transfusion that are nearing expiry. BID has been developed for the Central Zone-BTS (CZ-BTS) servicing Halifax, Canada.

In the recent years, there have been studies around real-time blood product inventory dashboards [2]-[4]. Sharpe et al. [4] developed a real-time dashboard that displays the Red Blood Cell (RBC) unit inventory in detail in a tabular format which is color-coded based on unit's closeness to expiry. Comparing the pre-implementation and postimplementation situations of the inventory proved that utilizing the dashboard reduced the outdate rates significantly. Gomez et al. [2] implemented a dashboard to manage a large platelet inventory, resulting in diminishing the outdate rates. Woo et al. [3] introduced a real-time dashboard suite for multiple blood products. We note that the existing blood inventory dashboards are cumbersome with limited interactive visualizations and filtering criterion, whilst lacking the ability to audit retrospective blood transfusion log to identify operational inefficiencies that lead to wastage due to discards. In this regard, our work advances the optimization of blood bank inventories from the various aspects, which has a direct impact on healthcare costs.

\section{BID design and implementation}

We take an IVA approach, where the real-time log (transaction data) representing the different states a blood unit experiences during its lifecycle is analyzed and visualized. Note that a blood unit goes through a number of transactional states, from its collection to transfusion/discard. Figure 1 shows the overall architecture of the BID. We collect real-time transactional data from the Laboratory Information System (LIS), which is a distributed system among all the blood transfusion sites. The backend is developed incorporating Java Spring framework to have a RESTful web service. The frontend is a combination of React and D3 which are JavaScript-based libraries, optimized for fast interactive multi-dimensional visualizations. The dashboard is updated every 30 minutes by sending an HTTPS GET request to the REST APIs - the update rate can be adjusted based on the transaction frequency in the blood product supply chain. 


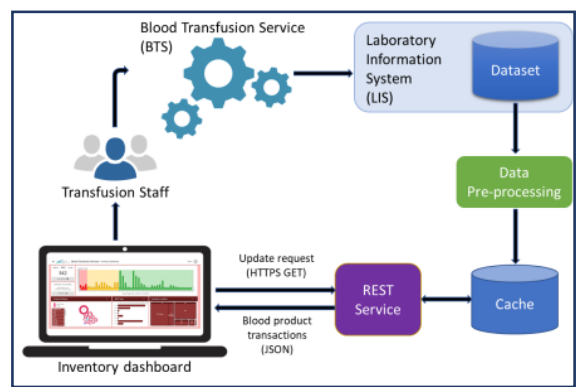

Figure 1. The functional architecture of BID

\section{BID Interface Design and Functionality}

BID interface (Figure 2) has a modular design, comprising interconnected information modules such that interactions in one module updates the information presented in others. At the top-level, Module 1 shows the current inventory level of the selected blood product; this number changes to show the inventory of specific units in user queries to find specific blood units. The view detail button presents information about all the selected units in terms of unit number, time to expiry, past transactional steps, product type, etc. To avoid outdates, Module 2 represents the inventory from the shelf-life viewpoint. Since avoiding outdates is central to inventory management, a heat-histogram is used to visualize the time-to-expiry of units which is divided into three color coded zones based on the number of days remaining to expiry-i.e., red for $\leq 3$ days warning about the units that are about to expire, orange for $\leq 15$ days and green for $>15$ days). Note that for RBC, the shelf-life of a blood unit is 42 days hence the heatmap shows a blood unit life in the range of 1-42 days. We implemented sliders within the heatmap visualization to enable staff to focus on specific expiry subperiods, such as examining only those units that have 10 days to expire. As the slider is used, the interconnected nature of the dashboard will update the information in all the other modules to focus on only the units in the selected subperiod. To facilitate crossmatching of blood units with the patient-specific transfusion requisition, the dashboard allows the generation of specialized multi-attribute queries by clicking and selecting the required attributes. Module 3 presents the distribution of the product attributes (e.g., units that are washed and have no negative antigen, units that have certain positive antigens and no other attributes, etc.). We used a space-efficient interactive combination of bubble-pie chart and priority list to facilitate query formulation. The priority list shows the attributes in the order of importance and can be adjusted according to the priorities of the staff. A combination of the antigens (positive and/or negative) and special typing can be selected via either the chart or the list to find units having all the selected attributes. To further facilitate crossmatching, Module 4 includes a histogram showing the number of units available for each blood type. The staff can click on multiple bars to apply the unit filtering based on the blood types. Module 5 illustrates the distribution of the units among inventory locations/sublocations. This is essential as most of the operational inefficiencies that lead to discard are caused by unnecessary unit transfers among transfusion sites. We utilized a treemap visualization to depict the blood unit volumes in different locations/sublocations in a space-efficient way-the greater the volume of units 
in a location/sublocation, the larger its representation on the treemap). Multiple inventory locations/sublocations can be selected via clicking on the treemap elements.

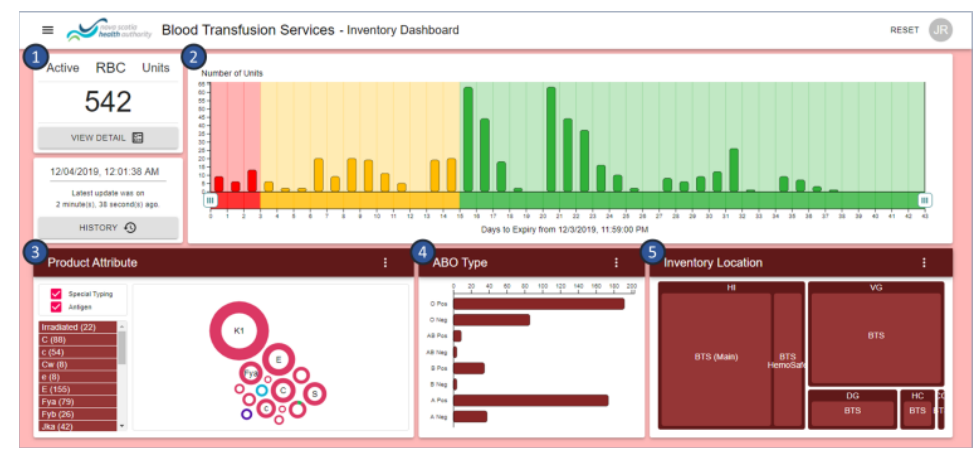

Figure 2. The modular design of the BID, illustrating the key modules

To illustrate the working of BID, we present a scenario that highlights the staff's interactions to inspect the inventory and find the right blood units. Since the O-negative blood type can be transfused urgently in the cases where crossmatching does not work out, the staff intends to see the O-negative RBC units that will be expired in the next 15 days, are in VG BTS inventory location and are irradiated. Figure 3 shows the BID interface as per the staff's interaction. It may be noted that 3 units are identified by the query; staff can explore the units' details via the view detail button (see Figure 4).

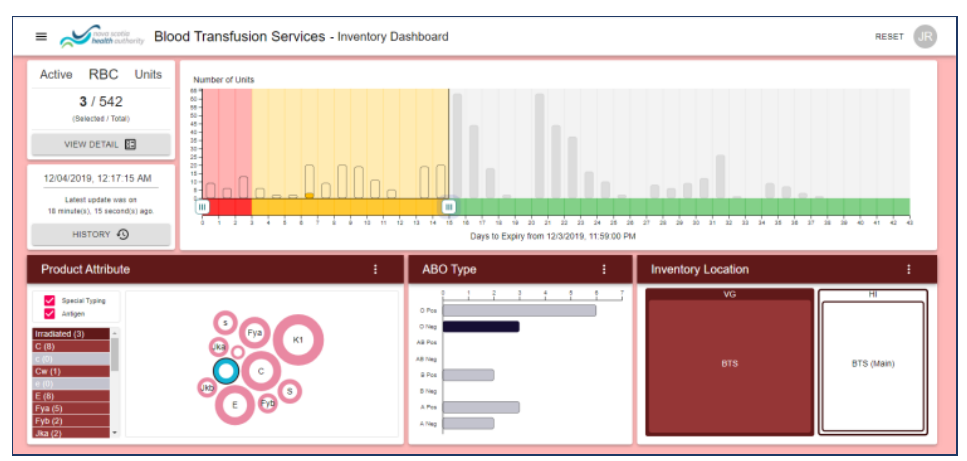

Figure 3. The dashboard interface after the staff interacted with all the modules.

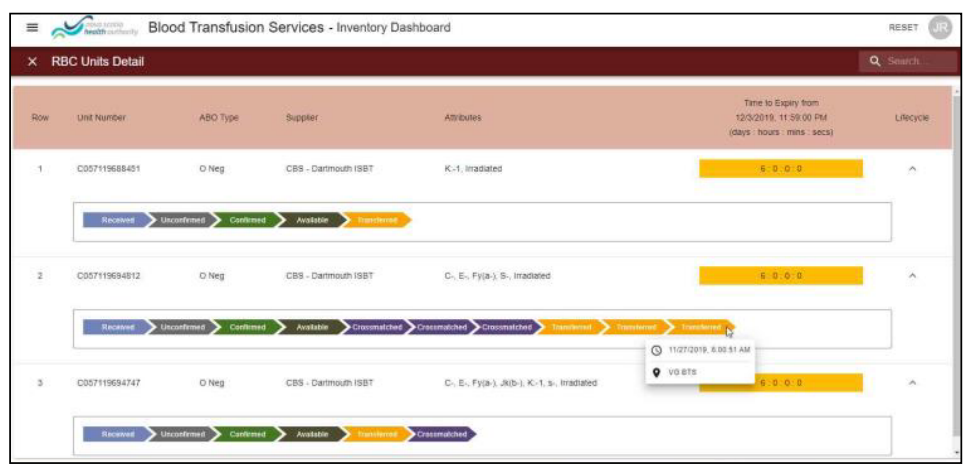

Figure 4. The list of the queried units that would be presented after clicking on the view detail button. 


\section{Concluding Remarks}

In our work, we investigated the use of interactive visual analytics to optimize inventory of blood banks by presenting a $360^{\circ}$ view of the inventory with associated services to select and prioritize blood units that both crossmatch with a transfusion request and are closest to expiry (to avoid wastage due to outdates). Our approach is innovative and effective, as current inventory management approaches are typically manual thereby resulting in high wastage and inefficient crossmatching practices. Interestingly, we take a novel blood unit lifecycle approach for the prioritization of a blood unit for transfusion, as opposed to just proximity to its expiry date-i.e., units that have been previously crossmatched and transferred to medical units and then returned back to the blood bank (some units experience this more than once) can now be tracked and prioritized for transfusion. Our web-based real-time dashboard plays an important role in three different yet interrelated aspects [5]: (1) Blood unit transaction data is complex as it is based on the LIS log, hence it is difficult to analyze and monitor. Our intuitive design of BID not only analyzes this complex data in real-time but makes it accessible for staff to have an overview of the blood bank thus facilitating operational decisions and selection of blood units for transfusion that minimize wastage; (2) The data visualization organizes the inventory information in purposeful modules thus allowing multiple views of the inventory and multiple dimensions for the selection of blood units; and (3) The multidimensional query formulation, by simply clicking on the visualization elements, is an effective feature as it allows the BTS staff to perform fine-grained crossmatching. Our visual query generator is dynamic and accumulative, whereby staff can select multiple attributes from different dimensions to formulate a query to crossmatch with the patient's specific transfusion requirements.

BID is designed to be scalable to any BTS. It is being implemented within CZ-BTS where it will handle live data streams. We plan an efficacy and usability evaluation study to investigate the impact of BID on BTS operations.

\section{Acknowledgement}

This research is supported by the Blood Efficiency Accelerator Award by Canadian Blood Services. We thank the NSHA Central Zone Blood Transfusion Services for providing us the dataset and supporting the project.

\section{References}

[1] Rad J, Cheng C, Quinn JG, Abidi S, Liwski R, Abidi SSR. An AI-Driven Predictive Modelling Framework to Analyze and Visualize Blood Product Transactional Data for Reducing Blood Products' Discards. in Lecture Notes in Computer Science, 2020, vol. 12299 LNAI, pp. 192-202, doi: 10.1007/978-3-030-59137-3 18.

[2] Gomez AT, Quinn JG, Doiron DJ, Watson S, Crocker BD, Cheng CK-W. Implementation of a novel real-time platelet inventory management system at a multi-site transfusion service. Transfusion. 2015; 55(9):2070-2075.

[3] Woo JS, et al. Development and Implementation of Real-Time Web-Based Dashboards in a Multisite Transfusion Service. J. Pathol. Inform., Feb 2019;10(3).

[4] Sharpe C, Quinn JG, Watson S, Doiron D, Crocker B, Cheng C. Novel web-based real-time dashboard to optimize recycling and use of red cell units at a large multi-site transfusion service. J. Pathol. Inform. 2014;5(1):35.

[5] Al-Kassab J, Ouertani ZM, Schiuma G, Neely A. Information visualization to support management decisions. Int. J. Inf. Technol. Decis. Mak. 2014;13(02):407-428. 